\title{
Activation of Receptors $\delta$ (PPAR $\delta$ ) by Agonist (GW0742) may Enhance Lipid Metabolism in Heart both In Vivo and In Vitro
}

Authors

Affiliations
S.-C. Kuo ${ }^{1,2 *}$, P.-M. Ku ${ }^{3,4 *}$, L.-J. Chen ${ }^{5}$, H.-S. Niu' ${ }^{6}$, J.-T. Cheng ${ }^{4,7}$

Affiliation addresses are listed at the end of the article
Key words

cardiomyocytes

- digoxin

fatty acid oxidation

- TCA cycle gene

- PPAR反 received 16.04.2013 accepted 21.05.2013

\section{Bibliography}

DOI http://dx.doi.org/ 10.1055/s-0033-1348317

Published online:

June 26, 2013

Horm Metab Res 2013;

45: 880-886

(c) Georg Thieme Verlag KG

Stuttgart · New York

ISSN 0018-5043

\section{Correspondence}

\section{J.-T. Cheng}

Institute of Medical Science

College of Health Science

Chang Jung Christian University

Guei-Ren

71101 Tainan City

Taiwan

Tel.: + 886/6/3318516

Fax: $+886 / 6 / 2386548$

jtcheng5503@yahoo.com.tw

\section{Abstract \\ $\nabla$}

It has been documented that cardiac agents may regulate the lipid metabolism through increased expression of PPARS in cardiac cells. However, the effect on lipid metabolism by direct activation of PPARS is still unknown. The present study applied specific PPARS agonist (GW0742) to investigate this point in the heart of Wistar rats and in the primary cultured cardiomyocytes from neonatal rat. Expressions of PPARS in the heart and cardiomyocytes after treatment with GW0742 were detected using Western blots. The fatty acid (FA) oxidation and the citric acid (TCA) cycle related genes in cardiomyocytes were

\section{Introduction}

$\nabla$

Peroxisome proliferator-activated receptors (PPARs) are ligand-activated transcriptional factors that regulate the expression of genes involved in lipid metabolism and inflammation [1]. Three subtypes of PPARs, including PPAR $\alpha$, PPAR $\gamma$, and PPARS, modulate the expressions of many genes to exert various bioactivities [1]. PPAR $\alpha$ is relatively abundant in tissues with a high oxidative capacity, such as liver and heart. PPAR $r$ expression is confined to a limited number of tissues, primarily adipose tissue $[1,2]$. The ubiquitously expressed PPAR $\delta$ enhances fatty acid catabolism in adipose tissue and muscle [1]. PPARS-dependent maintenance of inotropic function and metabolic effects is crucial for cardiomyocytes [3-5]. The activation of PPARS increases basal fatty acid (FA) oxidation to maintain the energy balance and cardiac function $[6,7]$. Many FA oxidation-related enzymes and mitochondrial respiratory uncoupling genes are regulated by PPAR $\delta$ in cardiomyocytes, such as pyruvate dehydrogenase kinase and acyl-CoA

${ }^{*}$ These authors contributed equally to this work. also examined. In addition, PPARS antagonist (GSK0660) and siRNA-PPARS were employed to characterize the potential mechanisms. After a 7-day treatment with GW0742, expressions of PPARS in the heart were markedly increased. Increased expressions of FA oxidation and TCA cycle related genes were also observed both in vivo and in vitro. This action of GW0742 was blocked by GSK0660 or by siRNA-PPARS. The obtained results show that activation of PPAR $\delta$ by GW0742 is responsible for the increase of FA oxidation and TCA cycle related genes in hearts. Role of PPARS in the regulation of lipid metabolism in heart is then established.

oxidase $1[8,9]$. Deletion of cardiac PPARS, which is accompanied by decreased contraction, increased left ventricular end-diastolic pressure, and lowered cardiac output, leads to decreased contraction and increased incidence of cardiac failure [3]. Various cardiac pathologies have been shown to involve decreased lipid metabolism and impaired ATP generation in heart failure [10-12], but it remains unknown whether PPARS is involved in the pathogenesis of cardiac disorders. Our previous study showed that cardiac agents improved cardiac contraction in STZ-diabetic rats is associated with a marked increase in cardiac PPARS expression [13]. Also, an increase of PPARS by digoxin is related to the regulation of FA oxidation genes [14].

GW0742 is a ligand of PPARs, which has 300-1000-fold selectivity for PPAR 8 vs. other PPARs [15], and shows full PPAR agonist-like action in cell cultures and animal models [1618]. It has been documented that activation of PPARS by GW0742 increases cardiac contractility in rats [19]. However, the effects on cardiac lipid metabolism by GW0742 remain unclear. In the present study, we used Wistar rats and primary 
Table 1 Real-time PCR primers and universal library probes (UPL) of target genes.

\section{Gene Name}

Acetyl-coenzyme A dehydrogenase, very long chain (VLCAD)

Pyruvate dehydrogenase kinase, isoenzyme 4 (PDK4)

Uncoupling protein 3 (UCP3)

Malonyl-coenzyme A decarboxylase (MCD)

Acetyl-coenzyme A dehydrogenase, long chain (LCAD)

Acetyl-coenzyme A oxidase 1, palmitoyl (ACOX1)

Hydroxymethylbilane synthase (HBMS)

\section{Forward}

ggtggtttgggectctcta gagctgttctcccgctacag cccctacactgtatgctgagg bbctgtgatggcgtatc gcagttacttgggaagagcaa caccttcgagggagagaaca tecctgaaggatgtgcctac

\section{Reverse}

gggtaacgctaacaccaagg

ttctctcacaggcattttctga

agaaaggagggcatgaatcc

gagctggtgaggcctttg

ggcatgacaatatctgaatgga

cgcacctggtcgtagatttt

aagggttttcccgtttgc
UPL Number

53

120

79

158

81

112

79 neonatal cardiomyocytes of rats to investigate the effects of GW0742 on cardiac FA oxidation and TCA cycle genes in relation to PPARS.

\section{Materials and Methods}

\section{Materials}

GW0742 (a specific PPARS agonist) and GSK0660 (a specific PPARS antagonist) were purchased from Santa Cruz Biotechnology, Inc. (Santa Cruz, CA, USA). The TRIzol RNA extraction reagent, Opti-MEM ${ }^{\circledR}$ I Reduced Serum Medium, Stealth ${ }^{\mathrm{TM}}$ Select RNAi (siRNA-PPARS), scramble siRNA (siRNA-control), and Lipofectamine $2000^{\mathrm{TM}}$ were from Invitrogen (Carlsbad, CA, USA). Antibodies to PPARS and actin were purchased from Abcam (Cambridge, MA, USA). The LightCycler TaqMan Master kit, primers, and universal library probes for analyzing PPARS, fatty acid oxidation and TCA cycle related genes ( $\bullet$ Table 1 ) were purchased from Roche Diagnostics Corp. (Mannheim, Germany).

\section{Animals}

The male Wistar rats, weighing from 200 to $250 \mathrm{~g}$, were obtained from the Animal Center of National Cheng Kung University Medical College. All experiments on rats were conducted under anesthesia with $3 \%$ isoflurane. All animal procedures were performed according to the Guide for the Care and Use of Laboratory Animals published by the US National Institutes of Health (NIH Publication No. 85-23, revised 1996).

\section{Drug administration}

Animals were randomly assigned into 3 groups: (I) the control group $(n=8)$ treated with the vehicle, saline $(0.9 \%$ sodium chloride, intravenously); (II) the GW0742 (GW) group ( $\mathrm{n}=8)$ treated with GW0742 at $5 \mathrm{mg} / \mathrm{kg}$, intravenously for 7 days as described previously [20], and (III) the GW0742+GSK0660 (GW + GSK) group $(\mathrm{n}=8)$ treated with GW0742 $(5 \mathrm{mg} / \mathrm{kg})$ and GSK0660 at effective dose (3 mg/kg) [21] intravenously for 7 days. At the end of experiment, hearts of each group were dissected for detections using Western blotting analysis and real-time reverse transcription-polymerase chain reaction.

\section{Cell culture and treatment}

Primary cultures of neonatal rat cardiomyocytes were prepared by modification of a previously described method [22]. Briefly, the heart tissue from a 1-2-day-old Wistar rat was cut into $1-2 \mathrm{~mm}$ pieces and predigested with trypsin to remove red blood cells. The heart tissue was then digested with $0.25 \%$ trypsin and $0.05 \%$ collagenase. The dissociated cells were placed in uncoated $10 \mathrm{~cm}$ dishes and incubated at $37^{\circ} \mathrm{C}$ in a $5 \% \mathrm{CO}_{2}$ incubator for at least $1 \mathrm{~h}$ to remove the nonmyocytic cells. This procedure caused most of the fibroblasts to attach to the dishes, while most of the cardiomyocytes remained unattached. The population of cells enriched in cardiomyocytes was then collected and counted. The cells were cultured in DMEM (GIBCO BRL, Gaithersburg, MD, USA) with $1 \mathrm{mmol} / 1$ pyruvate, $10 \%$ fetal bovine serum (FBS), 100 units/ml penicillin, and 100 units $/ \mathrm{ml}$ of streptomycin. On the second day after plating, the medium was replaced. 3 days after plating, the cells were exposed to hyperglycemic conditions as described in detail later. Animal handling and disposal were performed in accordance with NIH guidelines. The GW0742-treated cardiomyocytes were generated from the incubation of cells with GW0742 $\left(10^{-6} \mathrm{~mol} / \mathrm{l}\right.$; M) for 24h [23], and the PPARS silenced cardiomyocytes were also used for the same treatment with GW0742. After the treatment, cells were washed twice, and harvested by trypsinization. Then, cells were collected and subjected to real-time reverse transcriptionpolymerase chain reaction or Western blotting analysis.

\section{Western blotting analysis}

Similar to our previous report [13], protein was extracted from tissue homogenates or cell lysates using ice-cold RIPA buffer supplemented with phosphatase and protease inhibitors ( $50 \mathrm{mmol} / \mathrm{l}$ sodium orthovanadate, $0.5 \mathrm{mM}$ phenylmethylsulfonyl fluoride, $2 \mathrm{mg} / \mathrm{ml}$ aprotinin, and $0.5 \mathrm{mg} / \mathrm{ml}$ leupeptin). Protein concentrations were determined with the Bio-Rad protein assay (Bio-Rad Laboratories, Inc., Hercules, CA, USA). Total proteins $(30 \mu \mathrm{g})$ were separated by SDS/polyacrylamide gel electrophoresis (10\% acrylamide gel) using the Bio-Rad Mini-Protein II system. Protein was transferred to expanded polyvinylidene difluoride membranes (Pierce, Rockford, IL, USA) with a Bio-Rad Trans-Blot system. After transfer, the membranes were washed with PBS and blocked for $1 \mathrm{~h}$ at room temperature with $5 \%(\mathrm{w} / \mathrm{v})$ skimmed milk powder in PBS. The manufacturer's instructions were followed for the primary antibody reactions. Blots were incubated overnight at $4{ }^{\circ} \mathrm{C}$ with an immunoglobulin-G polyclonal rabbit anti-mouse antibody (Affinity BioReagents, Inc., Golden, CO, USA) (1:500) in 5\% (w/v) skimmed milk powder dissolved in PBS/Tween 20 (0.5\% by volume) to bind the PPARS in the heart specimens. The blot was incubated with goat polyclonal antibody (1:1000) to bind the actin serving as internal control. After the removal of primary antibody, the blots were extensively washed with PBS/Tween 20. The blots were then incubated for $2 \mathrm{~h}$ at room temperature with the appropriate peroxidase-conjugated secondary antibody diluted in $5 \%(\mathrm{w} / \mathrm{v})$ in skimmed milk powder and dissolved in PBS/Tween 20. The blots were developed by autoradiography using the ECL-Western blotting system (Amersham International, Buckinghamshire, UK). The immune blot of PPARS (49 kDa) was quantified with a laser densitometer. 

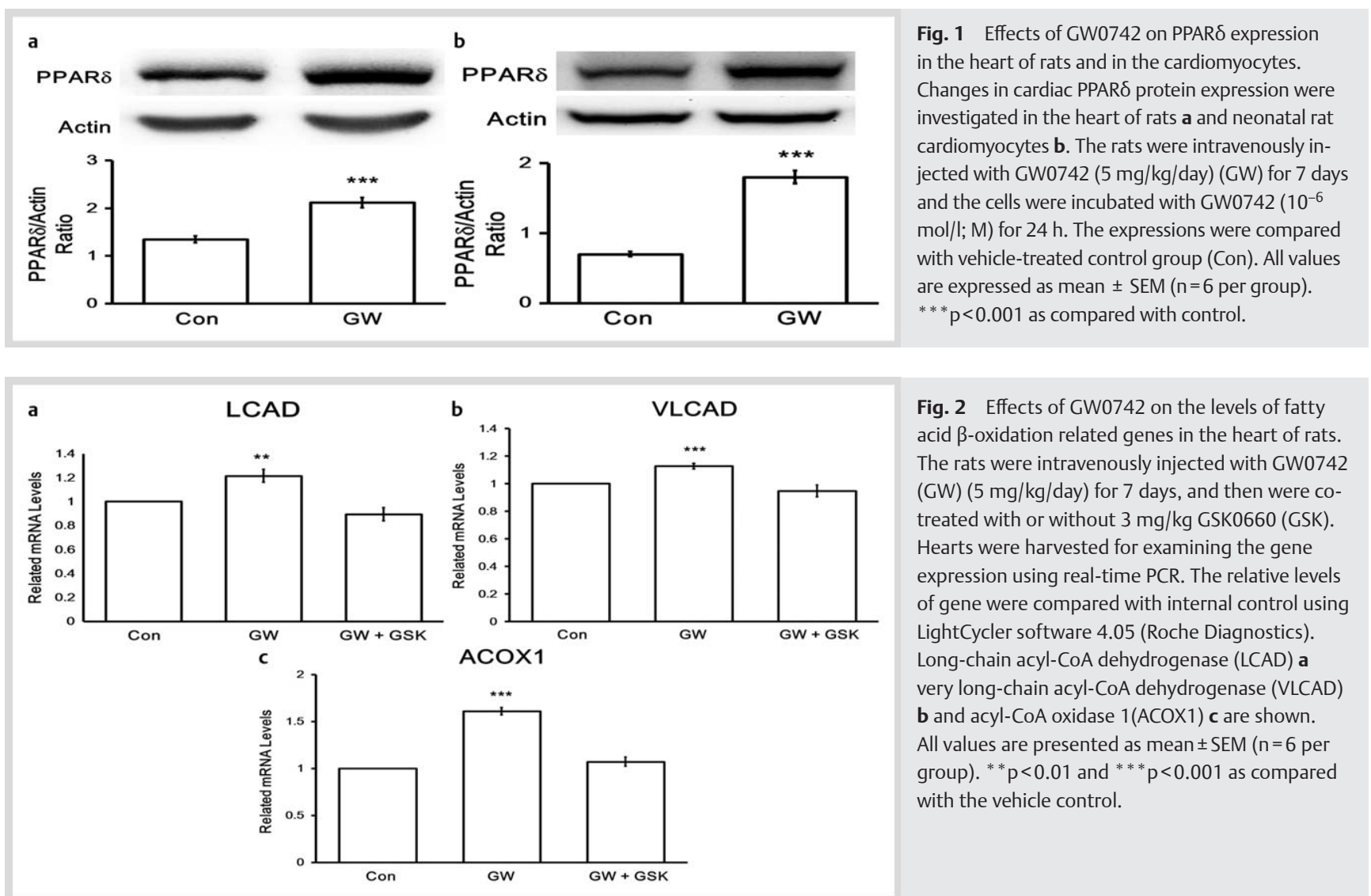

Fig. 2 Effects of GW0742 on the levels of fatty acid $\beta$-oxidation related genes in the heart of rats. The rats were intravenously injected with GW0742 (GW) $(5 \mathrm{mg} / \mathrm{kg} /$ day) for 7 days, and then were cotreated with or without $3 \mathrm{mg} / \mathrm{kg}$ GSK0660 (GSK). Hearts were harvested for examining the gene expression using real-time PCR. The relative levels of gene were compared with internal control using LightCycler software 4.05 (Roche Diagnostics). Long-chain acyl-CoA dehydrogenase (LCAD) a very long-chain acyl-CoA dehydrogenase (VLCAD) b and acyl-CoA oxidase 1(ACOX1) c are shown. All values are presented as mean \pm SEM $(n=6$ per group). ${ }^{* *} p<0.01$ and ${ }^{* * *} p<0.001$ as compared with the vehicle control.

Real-time reverse transcription-polymerase chain reaction Total RNA was extracted from heart ventricles and cell lysates with TRIzol reagent (Invitrogen Corp., Carlsbad, CA, USA). The web-based assay-design software from the Universal Probe Library Assay Design Center (http://www.roche-applied-sci ence.com/sis/rtpcr/upl/adc.jsp) was used to design TaqMan primer pairs and to select appropriate hybridization probes. All the PCR experiments were performed using a LightCycler (Roche Diagnostics $\mathrm{GmbH}$, Mannheim, Germany). Reactions were performed in $20 \mu \mathrm{l}$ of a mixture consisting of $13.4 \mu \mathrm{l}$ of PCR buffer, $0.2 \mu \mathrm{l}$ of each of the Universal Probe Library probes $(10 \mathrm{mmol} / \mathrm{l})$ ( $\odot$ Table 1 ), $0.2 \mu \mathrm{l}$ of each primer $(20 \mu \mathrm{mol} / \mathrm{l}), 4 \mu \mathrm{l}$ of LightCycler TaqMan Master (Roche Diagnostics $\mathrm{GmbH}$ ) and $2 \mu$ lof template cDNA. The thermal cycling conditions consisted of an initial denaturation step at $95^{\circ} \mathrm{C}$ for $10 \mathrm{~min}$, followed by 45 cycles at $94^{\circ} \mathrm{C}$ for $10 \mathrm{~s}, 60^{\circ} \mathrm{C}$ for $20 \mathrm{~s}$, and $72^{\circ} \mathrm{C}$ for $1 \mathrm{~s}$. The crossing point for each amplification curve was determined by the second derivative maximum method. The concentration of each gene was calculated by reference to the respective standard curve with the aid of the LightCycler software. Relative gene expression was expressed as the ratio of the concentration of the target gene to that of a housekeeping gene hydroxymethylbilane synthase (HBMS).

\section{Small interfering RNA (siRNA)}

Duplexed RNA oligonucleotides for rat PPARS (Stealth RNAi ${ }^{\mathrm{TM}}$ ) were synthesized from Invitrogen. According to our previous method [13], cardiomyocytes were transfected with $40 \mathrm{pmol}$ of PPARS-specific siRNAs (siRNA-PPARS) or scramble siRNA using Lipofectamine 2000 (Invitrogen) and they were treated $48 \mathrm{~h}$ post-transfection. The sequences of the siRNA-PPARS are UUGCAGAUCCGAUCGCACUUCUCGU (sense strand) and ACGAGAAGUGCGAUCGGAUCUGCAA (antisense strand).

\section{Statistical analysis}

Data are expressed as the mean \pm SEM for the number ( $\mathrm{n}$ ) of animals in 1 group as indicated. Statistical analysis was carried out using repeated measures analysis of variance (ANOVA) and Newman-Keuls post-hoc analysis. Bonferroni's correction was applied to the data, which were obtained from relatively small groups. A p-value of 0.05 or less was considered significant.

\section{Results \\ $\nabla$}

Effects of GW0742 on PPAR $\delta$ expression in the heart of rats and in the cardiomyocytes

The level of PPAR $\delta$ protein was significantly increased in the heart of rats, which received GW0742-treatment as compared with the control rats ( $\odot$ Fig. 1a). Also, a significant induction of the expression of PPARS protein was observed in GW0742treated neonatal rat cardiomyocytes ( $\bullet$ Fig. $1 \mathbf{b}$ ).

\section{Effects of GW0742 on the levels of fatty acid}

$\beta$-oxidation related genes in the heart of rats

We examined the transcription levels of fatty acid $\beta$-oxidation genes using real-time PCR [3]. The relative levels of gene expression were compared to the internal control with the LightCycler software 4.05. Long-chain acyl-CoA dehydrogenase (LCAD), very long-chain acyl-CoA dehydrogenase (VLCAD), and acyl-CoA oxidase 1 (ACOX1) were increased in the heart of GW0742-treated rats. As compared to the control rats treated with vehicle, the transcription levels were increased by $122 \%$ for LCAD, $113 \%$ for VLCAD, and $161 \%$ for ACOX1, respectively ( $\odot$ Fig. 2 ). In addition, effects of GW0742 on these genes were blocked by co-treatment with GSK0660 (๑ Fig. 2). 


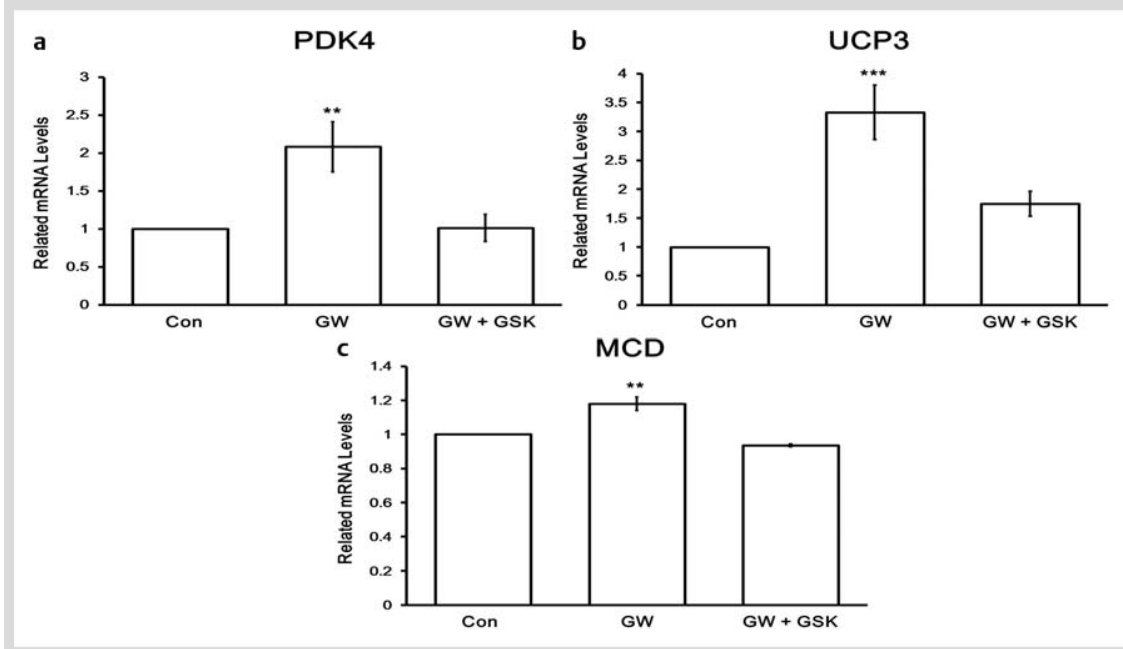

Fig. 3 Effects of GW0742 on the levels of TCA cycle related genes in the heart of rats. The rats were intravenously injected with GW0742 (GW) $(5 \mathrm{mg} /$ $\mathrm{kg} /$ day) for 7 days, and then were co-treated with or without $3 \mathrm{mg} / \mathrm{kg}$ GSK0660 (GSK). Hearts were harvested for examining the gene expression using real-time PCR. The relative levels of gene were compared with internal control using LightCycler software 4.05 (Roche Diagnostics). Pyruvate dehydrogenase kinase 4 (PDK4) a uncoupling protein 3 (UCP3) b and malonyl-CoA decarboxylase (MCD) c are shown. All values are presented as mean \pm SEM ( $n=6$ per group). ${ }^{* *} p<0.01$ and ${ }^{* * *} p<0.001$ as compared with the vehicle control.
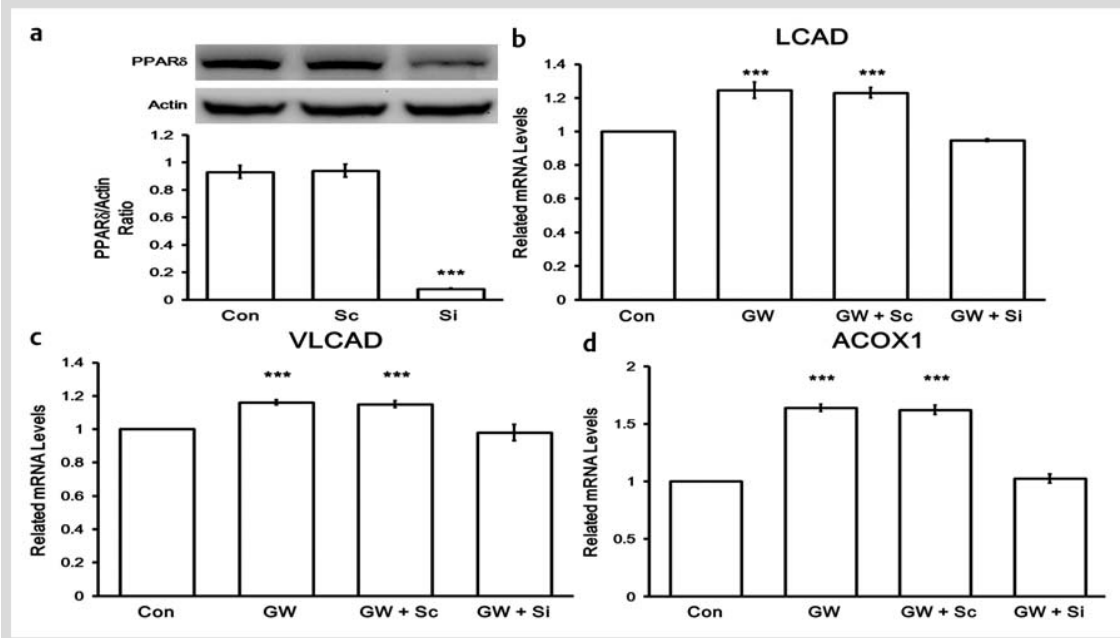

Fig. 4 Effects of GW0742 on the levels of fatty acid $\beta$-oxidation related genes in the neonatal rat cardiomyocytes. Cells were treated with siRNAPPARS (Si) or scramble siRNA (Sc) a Cells were incubated with GW0742 (10 $\left.10^{-6} \mathrm{~mol} / \mathrm{l} ; \mathrm{M}\right)$ (GW) for $24 \mathrm{~h}$ and were harvested for examining the gene expression by real-time PCR. The relative levels of gene were compared with internal control using LightCycler software 4.05. Long-chain acyl-CoA dehydrogenase (LCAD) b very long-chain acyl-CoA dehydrogenase (VLCAD) $\mathbf{c}$ and acyl-CoA oxidase 1 (ACOX1) $\mathbf{d}$ are shown. All values are presented as mean \pm SEM ( $n=6$ per group). ${ }^{* *} p<0.01$ and ${ }^{* * *} p<0.001$ as compared with the vehicle control.

\section{Effects of GW0742 on the levels of TCA cycle related genes in the heart of rats}

We examined the transcription levels of TCA cycle genes using real-time PCR [3]. The relative levels of gene expression were compared to the internal control with the LightCycler software 4.05. Uncoupling protein 3 (UCP3), malonyl-CoA decarboxylase (MCD) and pyruvate dehydrogenase kinase 4 (PDK4) were raised in the heart of GW0742-treated rats. As compared to control rats treated with vehicle, the transcription levels were increased by $208 \%$ for PDK4, 333\% for UCP3 and $118 \%$ for MCD, respectively ( $\odot$ Fig. 3). Also, effects of GW0742 on these genes were blocked by the co-treatment with GSK0660 ( 0 Fig. 3).

\section{Effects of GW0742 on the levels of fatty acid $\beta$-oxidation related genes in the neonatal rat cardiomyocytes}

The transcription levels of fatty acid $\beta$-oxidation genes were further examined in neonatal rat cardiomyocytes using real-time PCR [3]. The mRNA levels of long-chain acyl-CoA dehydrogenase (LCAD), very long-chain acyl-CoA dehydrogenase (VLCAD), and acyl-CoA oxidase 1 (ACOX1) were also raised in neonatal rat cardiomyocytes by GW0742. As compared to control group, the transcription levels were increased by $125 \%$ for LCAD, $116 \%$ for VLCAD and $164 \%$ for ACOX1, respectively ( $\odot$ Fig. 4). In addition, effects of GW0742 on these genes were reversed in cells transfected with PPAR $\delta$-specific siRNA ( $\bullet$ Fig. 4).
Effects of GW0742 on the levels of TCA cycle related genes in the neonatal rat cardiomyocytes

The transcription levels of TCA cycle genes were further examined in neonatal rat cardiomyocytes using real-time PCR [3]. The mRNA levels of uncoupling protein 3 (UCP3), malonyl-CoA decarboxylase (MCD), and pyruvate dehydrogenase kinase 4 (PDK4) were markedly raised in neonatal rat cardiomyocytes by GW0742. As compared to control rats treated with vehicle, the transcription levels were increased by $258 \%$ for PDK4, $298 \%$ for UCP3, and $121 \%$ for MCD, respectively ( $\bullet$ Fig. 5 ). Also, effects of GW0742 on these genes were blocked in cells transfected with PPAR $\delta$-specific siRNA ( $\odot$ Fig. 5 ).

\section{Discussion}

The present study showed that administration of GW0742 causes an increase of PPARS expression in the heart of rats and neonatal rat cardiomyocytes. We also demonstrated the fatty acid $\beta$-oxidation and TCA cycle related genes could be upregulated by GW0742 both in the heart of rats and neonatal rat cardiomyocytes. Furthermore, the increased expressions of these genes by GW0742 were suppressed by GSK0660 or siRNA-PPARS in the heart of rats or neonatal rat cardiomyocytes. Thus, it can be identified that an activation of PPAR $\delta$ by GW0742 is related to 


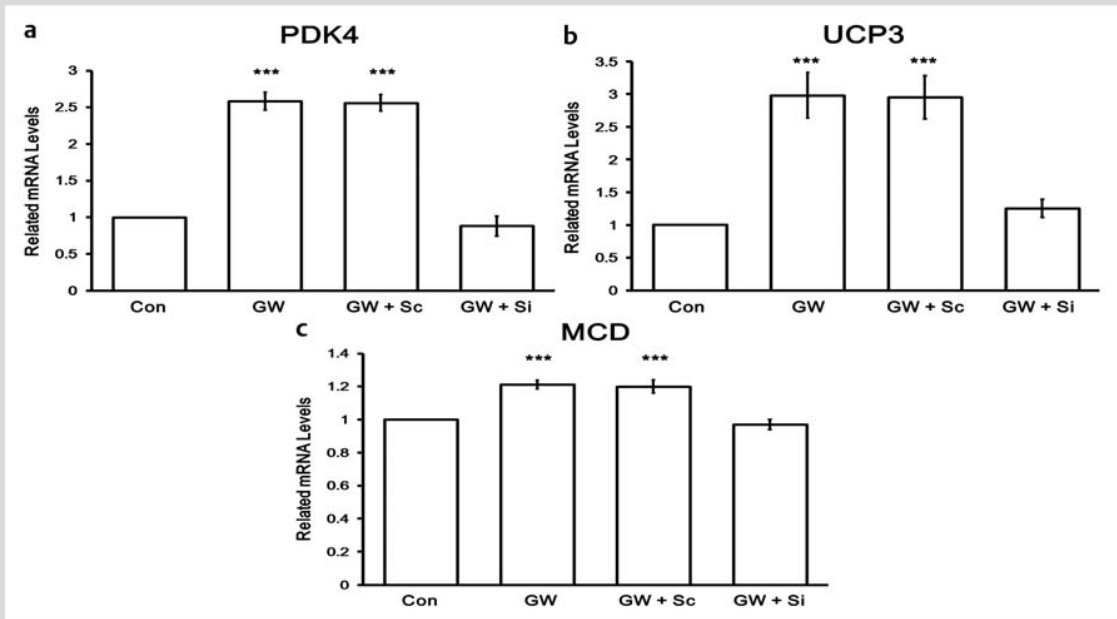

Fig. 5 Effects of GW0742 on the levels of TCA cycle related genes in the neonatal rat cardiomyocytes. Cells were incubated with GW0742 $\left(10^{-6}\right.$ $\mathrm{mol} / \mathrm{l} ; \mathrm{M}$ ) (GW) for $24 \mathrm{~h}$ and were harvested for examining the gene expression by real-time PCR. The relative levels of gene were compared with internal control using LightCycler software 4.05. Pyruvate dehydrogenase kinase 4 (PDK4) a uncoupling protein 3 (UCP3) b and malonyl-CoA decarboxylase $(M C D) c$ are shown. All values are presented as mean \pm SEM ( $n=6$ per group). ${ }^{* *} \mathrm{p}<0.01$ and ${ }^{* * *} p<0.001$ as compared with the vehicle control.

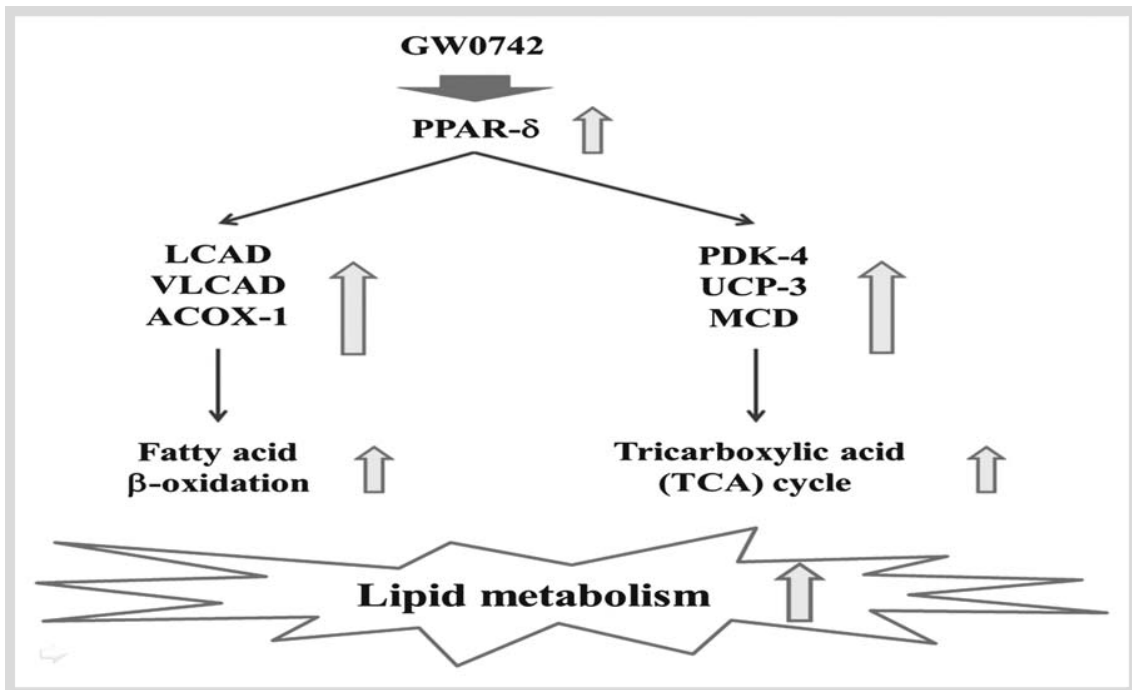

Fig. 6 Signal pathway of lipid metabolism enhanced by GW0742 in the heart of rats. Cardiac PPARס is activated by GW0742. Then, GW0742 has the ability in regulation of fatty acid (FA) metabolism via increased LCAD, VLCAD, ACOX-1 and PDK4, UCP-3, and MCD leading to the enhancement of $\beta$-oxidation and TCA cycle. the increase of lipid metabolism in heart; this view has not been mentioned before.

It has been established that PPAR $\delta$ plays an important role in the regulation of cardiac performance [24-26]. In our previous study, an activation of PPAR $\delta$ using the selective agonist GW0742 enhanced the cardiac contractility in isolated hearts and the hemodynamic $\mathrm{dP} / \mathrm{dt}_{\max }$ in rats; both actions of GW0742 were blocked by GSK0660 at a concentration sufficient to block PPAR $\delta$ $[27,28]$. In the present study, we have found that a 7-day treatment of GW0742 not only increased the level of PPARS expression but also upregulated the fatty acid $\beta$-oxidation and TCA cycle related genes in the hearts and cardiomyocytes. Moreover, PPARS activation in muscle is responsible for the lowering of plasma triglycerides in obese monkeys [29] and diabetic mice [30]. This supports the view that PPAR $\delta$ activation in muscle seems beneficial in the metabolism of lipids [31] by increasing the catabolism of lipids and decreasing lipid accumulation [31]. The enhancing effects of cardiac agent on lipid accumulation in heart appear to be related to the increased expression of PPAR $\delta$ [25]. In this report, we have demonstrated that GSK0660 and siRNA-PPARS suppressed the GW0742-induced actions regarding the increase in both expressions of PPARS and lipid metabolism related genes. These results suggest the mediation of PPAR $\delta$ in GW0742-induced actions for increased expressions of fatty acid $\beta$-oxidation and TCA cycle related genes in the heart.
The expression of PPARS is more ubiquitous, with relatively high levels in metabolically active tissues, such as muscle, liver, and adipose tissue [32]. Selective activation of PPARS by agonists has been shown to improve glucose metabolism and insulin sensitivity in mouse models of obesity and insulin resistance, and these results are mainly related to the agonists' capacity for activation of fatty acid transport and oxidation [33]. Activation of PPARS in skeletal muscle increases the expression of regulatory genes involved in FA metabolism and mitochondrial oxidative phosphorylation, such as CPT-1 and the uncoupling proteins $[34,35]$. In the present study, transfection with siRNA-PPARS suppressed the GW0742-induced PPARS expression and lipid metabolic genes in cardiomyocytes. Thus, it shows that expressions of FA oxidation and mitochondrial respiratory uncoupling genes are regulated by PPARS activated by GW0742.

PPARS activation reduced adiposity by decreasing intracellular triglyceride accumulation in mouse adipose tissue and liver. PPARS also enhanced $\beta$-oxidation in mouse preadipocytes [36]. PPARS mRNA is expressed at 10 and 50 times the concentrations of PPAR $\alpha$ and PPAR $\gamma$ mRA [32], respectively, in skeletal muscle, and administration of PPAR $\delta$ agonists results in an increase in expression of genes involved in fatty acid oxidation, mitochondrial respiration, and oxidative metabolism, decreasing muscle fatigability. In addition, activation of PPAR $\delta$ may increase the mitochondrial gene expression and function. It has been reported 
that TCA cycle related genes could be upregulated in skeletal muscle of rats after administration of PPARS agonist, such as acyl-CoA dehydrogenase long chain, acyl-CoA synthetase long-chain family member, carnitine palmitoyltransferase, inositol(myo)-1 (or 4)-monophosphatase, 2,4-dienoyl CoA reductase, hormone-sensitive lipase, high-density lipoprotein-binding protein, mitochondrial acyl-CoA thioesterase 1, pyruvate dehydrogenase kinase, peroxisomal D3,D2-enoyl-CoA isomerase, uncoupling protein, etc. [34]. Concordantly, PPARS transgenic mice display the enhanced exercise endurance as compared with the wild-type mice and showing more fatigue resistant of skeletal muscle Type I fibers $[37,38]$. In the current study, GW0742 increases the expression of TCA cycle related genes. Moreover, GSK0660 and siRNAPPARS suppressed this action of GW0742. Relation of PPAR $\delta$ with mitochondrial gene expression and function in heart can thus be considered. But it needs more investigations in the future.

In conclusion, expression of PPARS is raised by GW0742 in the heart and primary cultured rat cardiaomyocytes. Also, GW0742 increases lipid metabolism via an increase in LCAD, VLCAD, ACOX-1 and PDK-4, UCP-3, and MCD leading the enhancement of $\beta$-oxidation and TCA cycle as shown in 8 Fig. 6 by the direct activation of PPARS. These findings show that PPARS may play a role in the regulation of cardiac lipid metabolism and this is the first work showing the effects of GW0742 on metabolism of heart. Our previous study demonstrated that treatment of PPAR $\delta$ agonist could enhance cardiac contractility $[27,28]$. The metabolic activation in cardiac muscle may serve to supply the metabolic requirements for periods of increased physical load. Thus, PPARS agonist could be used as a good cardiac tonic agent in clinical application in the future.

\section{Conflicts of Interest}

\section{$\nabla$}

The authors declare that they have no conflicts of interest in the authorship or publication of this contribution.

\footnotetext{
Affiliations

${ }^{1}$ Department of Ophthalmology, Chi-Mei Medical Center, Yong Kang, Tainan City, Taiwan

${ }^{2}$ Department of Optometry, Chung Hwa University of Medical Technology, Jen-Teh, Tainan City, Taiwan

${ }^{3}$ Department of Cardiology, Chi-Mei Medical Center, Yong Kang, Tainan City, Taiwan

${ }^{4}$ Department of Medical Research, Chi-Mei Medical Center, Yong Kang, Tainan City, Taiwan

${ }^{5}$ Institute of Basic Medical Sciences, College of Medicine, National Cheng Kung University, Tainan City, Taiwan

Department of Nursing, Tzu Chi College of Technology, Hualien City, Taiwan

Department of Nutrition and Institute of Medical Science, College of Health Science, Chang Jung Christian University, Guei-Ren, Tainan City, Taiwan
}

\section{References}

1 Yang Q Li Y. Roles of PPARs on regulating myocardial energy and lipid homeostasis. J Mol Med 2007; 85: 697-706

2 Issemann I, Green S. Activation of a member of the steroid hormone receptor superfamily by peroxisome proliferators. Nature 1990; 347 : 645-650

3 Cheng L, Ding G, Qin Q, Huang Y, Lewis W, He N, Evans RM, Schneider $M D$, Brako FA, Xiao Y, Chen YE, Yang Q. Cardiomyocyte-restricted peroxisome proliferator-activated receptor-delta deletion perturbs myocardial fatty acid oxidation and leads to cardiomyopathy. Nat Med 2004; 10: 1245-1250
4 Cheng L, Ding G, Qin Q, Xiao Y, Woods D, Chen YE, Yang Q. Peroxisome proliferator-activated receptor delta activates fatty acid oxidation in cultured neonatal and adult cardiomyocytes. Biochem Biophys Res Commun 2004; 313: 277-286

5 Barish GD, Narkar VA, Evans RM. PPAR delta: a dagger in the heart of the metabolic syndrome. J Clin Invest 2006; 116: 590-597

6 Planavila A, Rodriguez-Calvo R, Jove M, Michalik L, Wahli W, Laguna JC, Vazquez-Carrera M. Peroxisome proliferator-activated receptor beta/ delta activation inhibits hypertrophy in neonatal rat cardiomyocytes. Cardiovasc Res 2005; 65: 832-841

7 Pesant M, Sueur S, Dutartre P, Tallandier M, Grimaldi PA, Rochette $L$, Connat JL. Peroxisome proliferator-activated receptor delta (PPARdelta) activation protects H9c2 cardiomyoblasts from oxidative stress-induced apoptosis. Cardiovasc Res 2006; 69: 440-449

8 Finck BN, Kelly DP. Peroxisome proliferator-activated receptor gamma coactivator-1 (PGC-1) regulatory cascade in cardiac physiology and disease. Circulation 2007; 115: 2540-2548

9 Rimbaud S, Garnier A, Ventura-Clapier R. Mitochondrial biogenesis in cardiac pathophysiology. Pharmacol Rep 2009; 61: 131-138

10 Ventura-Clapier R, Garnier A, Veksler V. Transcriptional control of mitochondrial biogenesis: the central role of PGC-1alpha. Cardiovasc Res 2008; 79: 208-217

11 Sack MN, Kelly DP. The energy substrate switch during development of heart failure: gene regulatory mechanisms (Review). Int J Mol Med 1998; $1: 17-24$

12 Bugger $H$, Abel ED. Molecular mechanisms for myocardial mitochondrial dysfunction in the metabolic syndrome. Clin Sci (Lond) 2008; 114: $195-210$

13 Yu BC, Chang CK, Ou HY, Cheng KC, Cheng JT. Decrease of peroxisome proliferator-activated receptor delta expression in cardiomyopathy of streptozotocin-induced diabetic rats. Cardiovasc Res 2008; 80: 78-87

14 Fan SC, Yu BC, Chen ZC, Chen LJ, Chung HH, Cheng JT. The decreased expression of peroxisome proliferator-activated receptors delta (PPARdelta) is reversed by digoxin in the heart of diabetic rats. Horm Metab Res 2010; 42: 637-642

15 Sznaidman ML, Haffner CD, Maloney PR, Fivush A, Chao E, Goreham D, Sierra ML, LeGrumelec C, Xu HE, Montana VG, Lambert MH, Willson $T M$, Oliver WR Jr., Sternbach DD. Novel selective small molecule agonists for peroxisome proliferator-activated receptor delta (PPARdelta) - synthesis and biological activity. Bioorg Med Chem Lett 2003; 13: 1517-1521

16 Bility MT, Devlin-Durante MK, Blazanin N, Glick AB, Ward JM, Kang BH, Kennett MJ, Gonzalez FJ, Peters JM. Ligand activation of peroxisome proliferator-activated receptor beta/delta (PPAR beta/delta) inhibits chemically induced skin tumorigenesis. Carcinogenesis 2008; 29: 2406-2414

17 Gaudel C, Schwartz C, Giordano C, Abumrad NA, Grimaldi PA. Pharmacological activation of PPARbeta promotes rapid and calcineurindependent fiber remodeling and angiogenesis in mouse skeletal muscle. Am J Physiol Endocrinol Metab 2008; 295: E297-E304

18 Wagner N, Jehl-Pietri C, Lopez P, Murdaca J, Giordano C, Schwartz C, Gounon P, Hatem SN, Grimaldi P, Wagner KD. Peroxisome proliferatoractivated receptor beta stimulation induces rapid cardiac growth and angiogenesis via direct activation of calcineurin. Cardiovasc Res 2009; 83: $61-71$

19 Chen ZC, Lee KS, Chen LJ, Wang LU, Niu HS, Cheng JT. Cardiac peroxisome proliferator-activated receptor d (PPARd) as a new target for increased contractility without altering heart rate. PloS one 2013 in press

20 Zarzuelo MJ, Jimenez R, Galindo P, Sanchez M, Nieto A, Romero M, Quintela AM, Lopez-Sepulveda R, Gomez-Guzman M, Bailon E, RodriguezGomez I, Zarzuelo A, Galvez J, Tamargo J, Perez-Vizcaino F, Duarte J. Antihypertensive effects of peroxisome proliferator-activated receptor-beta activation in spontaneously hypertensive rats. Hypertension 2011; 58: 733-743

21 Paterniti I, Esposito E, Mazzon E, Galuppo M, Di Paola R, Bramanti P, Kapoor A, Thiemermann C, Cuzzocrea S. Evidence for the role of peroxisome proliferator-activated receptor-beta/delta in the development of spinal cord injury. J Pharmacol Exp Therapeut 2010; 333: 465-477

22 Kannel WB, Hjortland M, Castelli WP. Role of diabetes in congestive heart failure: the Framingham study. Am J Cardiol 1974; 34: 29-34

23 Cohen G, Riahi Y, Shamni O, Guichardant M, Chatgilialoglu C, Ferreri C, Kaiser N, Sasson S. Role of lipid peroxidation and PPAR-delta in amplifying glucose-stimulated insulin secretion. Diabetes 2011; 60: 2830-2842

24 Chou MT, Lo SH, Cheng KC, Li YX, Chen LJ, Cheng JT. Activation of betaadrenoceptors by dobutamine may induce a higher expression of peroxisome proliferator-activated receptors delta (PPARdelta) in neonatal rat cardiomyocytes. Sci World J 2012; 2012: 248320. 
25 Chen ZC, Yu BC, Chen LJ, Cheng KC, Lin HJ, Cheng JT. Characterization of the mechanisms of the increase in PPARdelta expression induced by digoxin in the heart using the H9c2 cell line. Br J Pharmacol 2011; 163: 390-398

26 Chen ZC, Yu BC, Chen LJ, Cheng JT. Increase of Peroxisome Proliferator-activated Receptor delta (PPARdelta) by Digoxin to Improve Lipid Metabolism in the Heart of Diabetic Rats. Horm Metab Res 2013; 45: 364-371

27 Collino M, Benetti E, Miglio G, Castiglia S, Rosa AC, Aragno M, Thiemermann C, Fantozzi R. Peroxisome proliferator-activated receptor beta/ delta agonism protects the kidney against ischemia/reperfusion injury in diabetic rats. Free Radic Biol Med 2011; 50: 345-353

28 Kapoor A, Shintani Y, Collino M, Osuchowski MF, Busch D, Patel NS, Sepodes B, Castiglia S, Fantozzi R, Bishop-Bailey D, Mota-Filipe H, Yaqoob MM, Suzuki K, Bahrami S, Desvergne B, Mitchell JA, Thiemermann C. Protective role of peroxisome proliferator-activated receptor-beta/ delta in septic shock. Am J Respir Crit Care Med 2010; 182: 1506-1515

29 Schiaffino S, Serrano A. Calcineurin signaling and neural control of skeletal muscle fiber type and size. Trends Pharmacol Sci 2002; 23: 569-575

30 Hokanson JF, Mercier JG, Brooks GA. Cyclosporine A decreases rat skeletal muscle mitochondrial respiration in vitro. Am J Respir Crit Care Med 1995; 151: 1848-1851

31 Zhou L, Cabrera ME, Okere IC, Sharma N, Stanley WC. Regulation of myocardial substrate metabolism during increased energy expenditure: insights from computational studies. Am J Physiol Heart Circ Physiol 2006; 291: H1036-H1046
32 Braissant O, Foufelle F, Scotto C, Dauca M, Wahli W. Differential expression of peroxisome proliferator-activated receptors (PPARs): tissue distribution of PPAR-alpha, -beta, and -gamma in the adult rat. Endocrinology 1996; 137: 354-366

33 Takahashi S, Tanaka T, Sakai J. New therapeutic target for metabolic syndrome: PPARdelta. Endocr J 2007; 54: 347-357

34 Wang YX, Zhang CL, Yu RT, Cho HK, Nelson MC, Bayuga-Ocampo $C R$, Ham J, Kang H, Evans RM. Regulation of muscle fiber type and running endurance by PPARdelta. PLoS Biol 2004; 2: e294

35 Lin J, Wu H, Tarr PT, Zhang CY, Wu Z, Boss O, Michael LF, Puigserver P, Isotani E, Olson EN, Lowell BB, Bassel-Duby R, Spiegelman BM. Transcriptional co-activator PGC-1 alpha drives the formation of slowtwitch muscle fibres. Nature 2002; 418: 797-801

36 Wang YX, Lee CH, Tiep S, Yu RT, Ham J, Kang H, Evans RM. Peroxisomeproliferator-activated receptor delta activates fat metabolism to prevent obesity. Cell 2003; 113: 159-170

37 Luquet S, Lopez-Soriano J, Holst D, Fredenrich A, Melki J, Rassoulzadegan M, Grimaldi PA. Peroxisome proliferator-activated receptor delta controls muscle development and oxidative capability. FASEB J 2003; 17: 2299-2301

38 Kleiner S, Nguyen-Tran V, Bare $O$, Huang X, Spiegelman B, Wu Z. PPAR\{delta\} agonism activates fatty acid oxidation via PGC-1\{alpha\} but does not increase mitochondrial gene expression and function. J Biol Chem 2009; 284: 18624-18633 